\title{
Equidade na Utilização de Consultas Médicas em Portugal: Na saúde e na Doença, na Riqueza e na Pobreza?
}

\author{
Equity in Usage of Medical Appointments in Portugal: In \\ Sickness and in Health, in Poverty and in Wealth?
}

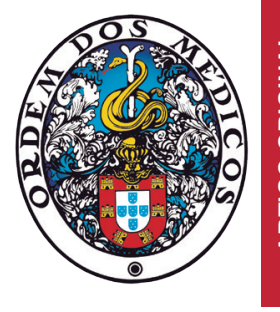

Carlota QUINTAL ${ }^{1}$, Micaela ANTUNES $\square^{1}$

Acta Med Port 2020 Feb;33(2):93-100 - https://doi.org/10.20344/amp.12278

\section{RESUMO}

Introdução: A equidade é um objetivo central da política de saúde em Portugal. Contudo, a evidência empírica sobre utilização de cuidados é escassa não existindo resultados atualizados. O nosso objetivo é avaliar o respeito pelo princípio de igual utilização para igual necessidade.

Material e Métodos: Usamos dados do Inquérito Nacional de Saúde 2014. A utilização de cuidados é medida pelo número de consultas de medicina geral e familiar ou de especialidades hospitalares. Para avaliar os fatores impactantes na utilização recorremos à análise de regressão multivariada (modelo binomial negativo). Para quantificar a desigualdade/iniquidade relacionada com o rendimento na utilização calcula-se o índice de concentração.

Resultados: Ter melhor saúde autoavaliada e não sofrer de limitações nas atividades diárias reduz a utilização; sofrer de doença crónica aumenta o uso. O rendimento não é estatisticamente significativo; a educação aumenta o uso, com efeito pronunciado. Viver em zonas urbanas e em Lisboa (comparado com o Norte) aumenta a utilização. Residir no Algarve ou Madeira, ou beneficiar apenas do Serviço Nacional de Saúde está associado a menos utilização. Pelo índice de concentração, não se exclui a hipótese de equidade nas consultas de medicina geral e familiar. Em relação às consultas de especialidades hospitalares e totais, a evidência sugere iniquidade favorável aos mais ricos.

Discussão: A iniquidade observada entre grupos de rendimento parece refletir desigualdades noutras variáveis de não-necessidade. Há a questão dos resultados poderem ser afetados por sobreutilização no caso de consultas de especialidades hospitalares.

Conclusão: Portugal progrediu favoravelmente em termos de equidade na utilização de consultas, mas subsistem desafios.

Palavras-chave: Acesso aos Serviços de Saúde; Disparidades em Assistência à Saúde; Disparidades nos Níveis de Saúde; Encaminhamento e Consulta; Equidade em Saúde; Portugal

\section{ABSTRACT}

Introduction: Equity is a central goal of health policy in Portugal. However, empirical evidence regarding healthcare usage is scarce and there is a lack of up-to-date results. Our objective is to evaluate whether the principle of equal utilisation for equal need has been met.

Material and Methods: We use data from the National Health Survey 2014. Healthcare usage is measured by the number of visits to a Family Physician or to a hospital-based specialist. To assess the factors affecting usage we adopted a multivariate regression analysis (Negative Binomial Model). To quantify income-related inequality/inequity in utilisation we computed the concentration index.

Results: Better self-assessed health and absence of limitations in daily activities decrease usage; suffering from chronic disease increases usage. Income is not statistically significant; education positively affects usage with a pronounced effect. Living in urban areas increases usage as well as living in Lisbon (compared to North). Living in Algarve or Madeira, or benefiting only from the National Health Service coverage negatively affects usage. The possibility for equity in Family Physician visits cannot be discarded. Regarding hospital based specialist and total visits, the evidence suggests the existence of pro-rich inequity.

Discussion: The observed income-related inequity seems to reflect inequalities in other non-need variables. Whether the results are affected by overuse, in the case of hospital based specialist visits, is an issue open to question.

Conclusion: Portugal evolved favourably in terms of equity in healthcare usage but several challenges remain.

Keywords: Health Equity; Health Services Accessibility; Health Status Disparities; Healthcare Disparities; Portugal; Referral and Consultation

\section{INTRODUÇÃO}

A equidade na utilização de cuidados de saúde é um princípio subscrito em muitos países, frequentemente vertido na sua legislação e documentos programáticos. ${ }^{1}$ Este é o caso de Portugal que desde logo na Constituição da República Portuguesa reconhece no seu Artigo $64 .^{\circ}$ a garantia do acesso dos cidadãos aos cuidados de saúde independentemente da sua condição económica. Garantias desta natureza surgem também na lei que cria o Serviço Nacional de Saúde, em 1979, e mais tarde na Lei de Bases da Saúde, em 1990. Em termos programáticos o reconhecimento da importância da equidade no acesso aos cuidados de saúde está inequivocamente espelhado num dos quatro eixos estratégicos, 'Equidade e Acesso Adequado aos Cuidados de Saúde', do Plano Nacional de Saúde em vigor até $2020 .{ }^{2}$ Neste contexto torna-se pertinente monitorizar este acesso, avaliando sucessos, eventuais falhas e áreas a melhorar.

O acesso é um conceito multidimensional envolvendo a interação entre fatores do lado da procura e do lado da oferta. ${ }^{3,4}$ Segundo o modelo de Andersen, ${ }^{3}$ a utilização depende da necessidade, mas é também condicionada por fatores predisponentes e fatores de capacitação. A necessidade

1. Centro de Investigação em Economia e Gestão (CeBER - Centre for Business and Economics Research). Faculdade de Economia. Universidade de Coimbra. Coimbra. Portugal.

$\triangle$ Autor correspondente: Micaela Antunes. micaela@fe.uc.pt

Recebido: 02 de maio de 2019 - Aceite: 03 de julho de 2019 | Copyright @ Ordem dos Médicos 2020 
é por regra associada ao estado de saúde, enquanto os fatores predisponentes incluem variáveis que influenciam a propensão dos indivíduos para procurarem cuidados, sejam sociodemográficas ou as crenças e atitudes em relação à saúde/doença. Os fatores de capacitação, por seu lado, incluem recursos pessoais, familiares ou comunitários, os quais podem facilitar ou dificultar a utilização de cuidados de saúde. A utilização de cuidados depende assim de várias dimensões inter-relacionadas como sejam a procura adequada de serviços, a disponibilidade, a proximidade, os custos diretos, os custos indiretos, a qualidade e a aceitação. ${ }^{5}$ Existindo utilização de cuidados (acesso realizado), a equidade no acesso tem sido interpretada em termos de igualdade de utilização para iguais necessidades, correspondendo esta abordagem à equidade horizontal na utilização. ${ }^{6,7} \mathrm{O}$ objetivo deste estudo é pois avaliar a equidade na utilização de cuidados de saúde, em concreto, na utilização de consultas médicas, recorrendo para tal ao Inquérito Nacional de Saúde de 2014 (INS 2014). Tanto quanto é do nosso conhecimento não existe evidência atualizada sobre este tema, nomeadamente com dados do último Inquérito Nacional de Saúde.

A literatura empírica sobre equidade na utilização de consultas médicas em Portugal é relativamente escassa, mas os resultados têm sido consistentes, apontando para a existência de fatores para além da necessidade clínica com influência sobre aquela utilização. Resultados com base em dados de $1996,{ }^{8}$ bem como com base em dados de $2000^{9}$ e de 1995 a $2001^{10}$ evidenciam iniquidade a favor dos grupos de maior rendimento, em particular no caso de consultas de especialidades hospitalares em comparação com consultas de medicina geral e familiar. De facto, no estudo envolvendo 21 países da OCDE, ${ }^{9}$ Portugal surge mesmo com o maior nível de iniquidade no caso das consultas das especialidades hospitalares. A evidência prévia sugere ainda a existência de iniquidade a favor dos indivíduos com nível educacional mais elevado. ${ }^{11,12}$ Para o grupo dos idosos, um estudo com base em dados de $2010^{13}$ encontrou evidência de iniquidade favorável aos mais ricos, quer no uso de consultas de medicina geral e familiar quer na variedade de especialistas consultados. Com este estudo pretendemos avaliar em que medida persistem estes padrões de utilização ou se pelo contrário o país evoluiu no sentido de maior igualdade na utilização para iguais necessidades.

\section{MATERIAL E MÉTODOS}

Os dados usados neste artigo são extraídos do quinto Inquérito Nacional de Saúde (2014). A base de amostragem são alojamentos em território nacional, e as unidades estatísticas de observação são os indivíduos, sendo entrevistado um único individuo por alojamento. Todos os procedimentos para a construção do inquérito, seleção da amostra, metodologia de entrevista, armazenamento de dados e verificação de coerência foram realizados de modo a assegurar a validade e representatividade dos dados. ${ }^{14}$

Para medir a utilização de cuidados de saúde, recorreu-se a duas questões, relativas, respetivamente, (i) à consulta com um médico de medicina geral e familiar nas últimas quatro semanas e (ii) à consulta com um médico de uma especialidade hospitalar nas últimas quatro semanas. De referir que apenas responderam a estas perguntas os indivíduos que numa questão prévia tinham respondido que a última consulta com o médico (de medicina geral e familiar ou de uma especialidade hospitalar, consoante o caso) ocorreu há menos de 12 meses. Assim, apenas estes indivíduos foram considerados no nosso estudo. A partir destas questões, criou-se uma variável adicional para captar o número total de consultas nas últimas quatro semanas, quer sejam de medicina geral e familiar ou de especialidade hospitalar.

Para avaliar os fatores impactantes na utilização de consultas médicas recorremos à análise de regressão multivariada, considerando variáveis de necessidade $(x)$ e variáveis de não-necessidade (z) (ver Apêndice 1: https:// www.actamedicaportuguesa.com/revista/index.php/amp/ article/view/12278/Apendice_01.pdf).

Uma vez que a variável 'número de consultas' assume valores inteiros, não-negativos e sem limite superior definido, sendo a respetiva distribuição caracterizada por muitos zeros e caudas grandes, modelos como o binomial negativo, que têm em conta a natureza não linear da análise, são habitualmente utilizados ${ }^{8,13}$ e é o adotado neste estudo. A justificação para este tipo de modelo prende-se com a existência de sobredispersão, que viola assim a hipótese de equidispersão do modelo de Poisson (média igual à variância) e permite evitar a obtenção de estimadores consistentes mas enviesados. A relação entre a variável de utilização $(y)$ e as variáveis de necessidade $(x)$ e de não-necessidade $(z)$ assume, nestas circunstâncias, uma especificação não-linear, expressa de forma genérica pela função $G$, na equação (1) ${ }^{1}$ :

$$
\mathrm{y}_{i}=G\left(\mathrm{a}+\sum_{j} \beta_{j} x_{j i}+\sum_{k} \gamma_{k} z_{k i}\right)+\varepsilon_{i}
$$

\section{[equação (1)]}

A variável dependente, $y_{i}$, representa a proxy para a utilização de cuidados de saúde do indivíduo $i$. As variáveis de não-necessidade $\left(z_{k}\right)$ são variáveis de controlo, incluídas na estimação para evitar enviesamento dos estimadores de necessidade $\left(x_{j}\right)$, por omissão de variáveis. Os parâmetros $\alpha, \beta_{\mathrm{j}}$ e $\gamma_{\mathrm{k}}$ são estimados pelo modelo binomial negativo, tendo sido utilizados desvios-padrão robustos. Em termos da interpretação dos resultados, qualquer impacto significativo de variáveis de não-necessidade sobre a utilização indicia a violação do princípio de igual utilização para igual necessidade.

Para quantificar as desigualdades relacionadas com o rendimento na utilização de consultas médicas recorre-se ao cálculo do índice de concentração. ${ }^{1,15} \mathrm{O}$ índice de concentração deriva da curva de concentração, que compara a percentagem acumulada da população, ordenada pelo rendimento (eixo das abcissas), com a percentagem acumulada da utilização (eixo das ordenadas). Quando este índice é nulo, existe igual utilização, quando é negativo (positivo), a utilização encontra-se desproporcionalmente concentrada 
nos mais pobres (ricos). O seu cálculo segue a proposta da literatura ${ }^{1}$ para o caso de dados agrupados (justificada pelo facto do INS 2014 apenas disponibilizar informação sobre o quintil de rendimento).

Contudo, e uma vez que a desigualdade no uso pode ser, pelo menos em parte, explicada pela desigualdade na necessidade, para a análise da equidade torna-se necessário calcular a utilização padronizada pela necessidade [equação (2)]. , $13^{13}$ A utilização padronizada pela necessidade, $\hat{y}_{i}^{I S}$, é assim dada pela diferença entre a utilização efetiva e a utilização prevista pela necessidade, adicionada do valor médio desta, sendo o tamanho da amostra dado por $n$ :

$$
\hat{\mathrm{y}}_{i}^{I S}=y_{i}-G\left(\hat{\mathrm{a}}+\sum_{j} \hat{\beta}_{j} x_{j i}+\sum_{k} \hat{\gamma}_{k} z_{k}\right)+1 / n \sum_{i=1}^{n} G\left(\hat{\mathrm{a}}+\sum_{j} \hat{\beta}_{j} x_{j i}+\sum_{k} \hat{\gamma}_{k} \bar{z}_{k}\right)
$$$$
\text { [equação (2)] }
$$

Após calcular a utilização padronizada, é possível obter o respetivo índice de concentração, designado de índice de iniquidade horizontal. Se o que diferencia a utilização dos indivíduos é a sua necessidade, os dois primeiros termos do membro direito da equação (2) anulam-se e os indivíduos apresentam todos a mesma utilização. Assim, se a utilização de cuidados for feita de acordo com a necessidade, o índice de iniquidade é nulo; caso o seu valor seja positivo, estamos na presença de iniquidade horizontal a favor dos mais ricos; se o índice for negativo, os indivíduos nos quintis de rendimento inferiores são os mais beneficiados.

No que diz respeito às questões éticas, o presente estudo utilizou dados provenientes do INS 2014, sem recolha de nova informação. Este inquérito foi conduzido pelo Instituto Nacional de Estatística e Instituto Nacional de Saúde Doutor Ricardo Jorge, sendo a recolha, tratamento e divulgação dos dados feita de acordo com o estabelecido pela Lei 22/2008 de 13 de maio, designadamente o artigo sexto que estabelece a aplicação do princípio do segredo estatístico aos dados. ${ }^{14}$

\section{RESULTADOS}

Relativamente ao painel de indivíduos considerado nas consultas de medicina geral e familiar, $40 \%$ são do sexo feminino, $36 \%$ tem 65 ou mais anos de idade e $81 \%$ considera o seu estado de saúde como sendo, no mínimo, razoável (ver Apêndice 2: https://www.actamedicaportuguesa.com/ revista/index.php/amp/article/view/12278/Apendice_02. pdf). Ainda assim, 68\% apresenta um problema de saúde prolongado, embora mais de metade dos indivíduos (54\%) não se sinta limitada nas suas atividades diárias devido a questões de saúde. Por outro lado, $58 \%$ dos indivíduos revela ter excesso de peso ou encontrar-se numa situação de obesidade. O número médio de doenças crónicas apontadas é dois (até um máximo de 12). Quanto às variáveis de não-necessidade, a distribuição por quintil de rendimento não revela grandes diferenças, sendo ligeiramente inferior a percentagem de indivíduos de rendimentos mais elevados (17\%); o mesmo se pode concluir sobre o tipo de área em que o indivíduo reside, com $38 \%$ a indicar residência numa zona pouco povoada. A maioria dos inquiridos (58\%) tem o ensino básico, ou seja, no máximo, terá o nono ano de escolaridade. Quase um quarto dos indivíduos vive sozinho e cerca de $40 \%$ encontra-se empregado. Registámos ainda que $60 \%$ dos indivíduos são casados ou vivem em união de facto e cerca de $70 \%$ não usufrui de qualquer subsistema ou seguro de saúde. Cerca de um quinto dos entrevistados é da região Centro.

Comparando as estatísticas descritivas anteriores com as que se referem a consultas de especialidades hospitalares, não se vislumbram grandes diferenças, com algumas exceções em variáveis de não-necessidade, como por exemplo o facto de a proporção de indivíduos com níveis de escolaridade mais elevados ser agora maior, com $34 \%$ a apresentar pelo menos o nível secundário. Por outro lado, o peso da ruralidade é agora ligeiramente menor, com cerca de $34 \%$ dos indivíduos a viver numa zona fracamente povoada. Encontram-se empregados $41 \%$ dos indivíduos (ligeiramente acima dos 39\% encontrados anteriormente) e $62 \%$ tem apenas o Serviço Nacional de Saúde - SNS (o que contrasta com os $68 \%$ do painel anterior, apesar de ainda assim representar uma expressão significativa).

O quadro relativo às estatísticas descritivas para o total de consultas não apresenta características distintivas dos anteriores.

No que diz respeito à análise de regressão, os resultados das estimações mostraram que a média condicional se apresenta como inferior à variância condicional (sobredispersão), para as três variáveis dependentes relativas ao uso de cuidados de saúde. Deste modo, confirma-se que a utilização do modelo binomial negativo é adequada. ${ }^{8,13}$

Relativamente aos fatores impactantes na utilização de consultas médicas apresentam-se, na Tabela 1 e na Fig. 1 , os efeitos marginais médios associados às variáveis explicativas utilizadas. Na Fig. 1 representam-se apenas os efeitos significativos a $1 \%$ e $5 \%$, sendo que na Tabela 1 a informação se apresenta de forma mais detalhada.

No caso das variáveis de necessidade, os efeitos são conforme o esperado. Considerar o estado de saúde como sendo razoável ou bom/muito bom diminui a utilização de todas as consultas, comparado com o estado de saúde $\mathrm{mau} / \mathrm{muito}$ mau. Na mesma linha, não ter limitações, ou ter em grau menor, diminui a utilização, comparado com ter muitas limitações nas atividades diárias. Em sentido contrário, sofrer de um problema prolongado e ter doença crónica aumenta o uso, embora neste caso as variáveis não sejam significativas para consultas de especialidades hospitalares. O excesso de peso e a idade apresentam sinais mistos não sendo, contudo, significativos. No caso da variável sexo, ser mulher implica menos utilização embora o efeito seja inferior a várias outras variáveis de necessidade e também não é significativo nas consultas de especialidades hospitalares.

No que diz respeito às variáveis de não-necessidade, de forma algo surpreendente, o rendimento não é estatisticamente significativo. Já no caso da educação, para consultas de especialidades hospitalares e totais, o efeito é 
Tabela 1 - Efeitos marginais médios das variáveis de necessidade e de não-necessidade, para cada proxy de utilização

\begin{tabular}{|c|c|c|c|c|c|c|}
\hline \multirow{2}{*}{$\begin{array}{l}\text { Variável } \\
\text { Variáveis de necessidade }\end{array}$} & \multicolumn{2}{|c|}{$\begin{array}{c}\text { cons_med_g } \\
\text { (1) }\end{array}$} & \multicolumn{2}{|c|}{$\underset{\text { (2) }}{\text { cons_espec }}$} & \multicolumn{2}{|c|}{$\begin{array}{c}\text { cons_total } \\
(3)\end{array}$} \\
\hline & & & & & & \\
\hline mulher & $-0,0276$ & * & $-0,0137$ & & $-0,0562$ & $* * *$ \\
\hline idade_15_24 & 0,0333 & & 0,0076 & & 0,0497 & \\
\hline idade_25_34 & 0,0181 & & $-0,0165$ & & 0,0319 & \\
\hline idade_35_44 & 0,0001 & & 0,0354 & & 0,0506 & \\
\hline idade_45_54 & 0,0106 & & 0,0184 & & 0,0299 & \\
\hline idade_55_64 & $-0,0054$ & & $-0,0255$ & & $-0,0191$ & \\
\hline idade_65_74 & 0,0442 & & $-0,0419$ & & 0,0285 & \\
\hline idade_75_84 & 0,0371 & & $-0,0446$ & & 0,0200 & \\
\hline esa_mb_b & $-0,1377$ & $* * *$ & $-0,2075$ & $* * *$ & $-0,2977$ & $* * *$ \\
\hline esa_r & $-0,0624$ & $* * *$ & $-0,1205$ & $* * *$ & $-0,1497$ & $* * *$ \\
\hline probl_prol & 0,0428 & $* *$ & 0,0354 & & 0,0885 & $* * *$ \\
\hline sem_lim & $-0,1955$ & $* * *$ & $-0,2417$ & $* * *$ & $-0,3487$ & $* * *$ \\
\hline pouco_lim & $-0,1095$ & $* * *$ & $-0,1551$ & $* * *$ & $-0,2077$ & $* * *$ \\
\hline mod_lim & $-0,0837$ & $* * *$ & $-0,0645$ & $* *$ & $-0,1177$ & $* * *$ \\
\hline exc_peso & 0,0009 & & $-0,0210$ & & $-0,0154$ & \\
\hline num_d_cron & 0,0090 & $* *$ & $-0,0034$ & & 0,0133 & $* *$ \\
\hline \multicolumn{7}{|c|}{ Variáveis de não-necessidade } \\
\hline Q2 & 0,0179 & & $-0,0113$ & & 0,0234 & \\
\hline Q3 & 0,0140 & & $-0,0355$ & & 0,0086 & \\
\hline Q4 & 0,0073 & & $-0,0445$ & & 0,0082 & \\
\hline Q5 & 0,0140 & & $-0,0056$ & & 0,0510 & \\
\hline bas & $-0,0151$ & & 0,0737 & ** & 0,0507 & * \\
\hline sec & $-0,0038$ & & 0,1641 & $* * *$ & 0,1373 & $* * *$ \\
\hline sup & $-0,0370$ & & 0,1897 & $* * *$ & 0,1375 & $* * *$ \\
\hline urbana & 0,0347 & * & 0,0848 & $* \star *$ & 0,0897 & *** \\
\hline zona_mista & $-0,0110$ & & 0,0375 & * & 0,0165 & \\
\hline vive_so & 0,0134 & & 0,0224 & & 0,0304 & \\
\hline agreg_mono & 0,0752 & * & 0,0570 & & 0,1122 & $* *$ \\
\hline agreg_casals & $-0,0117$ & & 0,0012 & & $-0,0011$ & \\
\hline emp & 0,0003 & & $-0,0519$ & * & $-0,0481$ & * \\
\hline desemp & 0,0238 & & $-0,0261$ & & $-0,0077$ & \\
\hline casado & 0,0079 & & 0,0184 & & 0,0340 & \\
\hline viúvo & $-0,0119$ & & $-0,0163$ & & $-0,0101$ & \\
\hline divorciado & $-0,0009$ & & $-0,0460$ & & $-0,0116$ & \\
\hline so_sns & $-0,0019$ & & $-0,0787$ & $* * *$ & $-0,0860$ & $* * *$ \\
\hline Algarve & $-0,0273$ & & $-0,0282$ & & $-0,0677$ & $* *$ \\
\hline Centro & $-0,0090$ & & $-0,0087$ & & $-0,0314$ & \\
\hline Lisboa & 0,0203 & & 0,0984 & $* * *$ & 0,0750 & $* *$ \\
\hline Alentejo & 0,0407 & * & 0,0448 & & 0,0442 & \\
\hline Açores & $-0,0193$ & & 0,0141 & & $-0,0524$ & \\
\hline Madeira & $-0,0583$ & $* *$ & $-0,0046$ & & $-0,0827$ & $* * *$ \\
\hline
\end{tabular}

${ }^{* * *} p<0,01 ;{ }^{* *} p<0,05 ;{ }^{*} p<0,1$

cons_med_g - $\mathrm{N}^{\circ}$ de consultas com médico de medicina geral e familiar nas últimas 4 semanas; cons_espec - $\mathrm{N}^{\circ}$ de consultas com médico de uma especialidade hospitalar nas últimas 4 semanas; cons_total - No de consultas com médico de medicina geral e familiar ou médico de uma especialidade hospitalar últimas 4 semanas

significativo e pronunciado, com os indivíduos com maior nível de escolaridade a apresentarem mais utilização de consultas. Outros resultados a destacar dizem respeito ao efeito de residir em zona urbana (maior utilização do que 


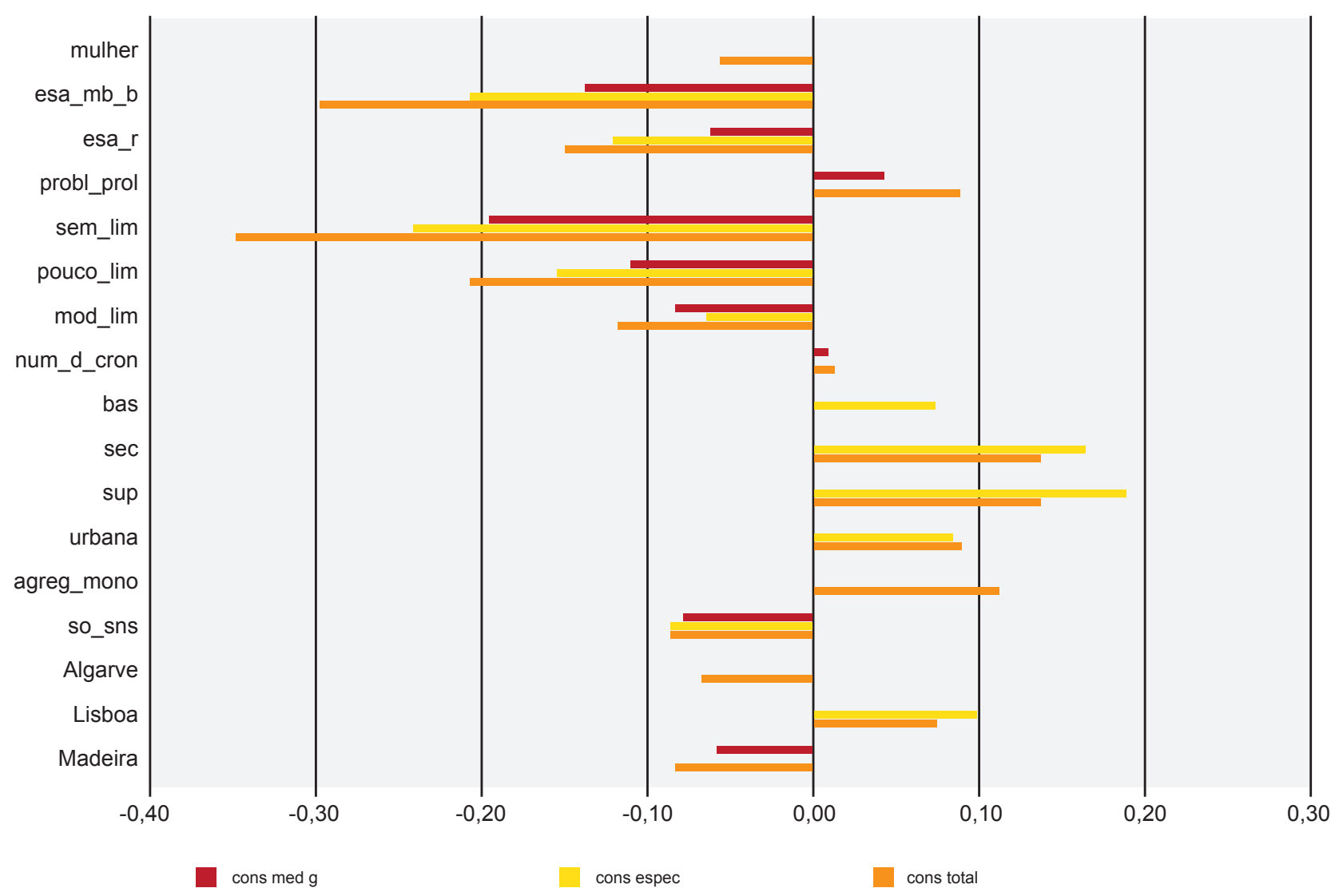

Figura 1 - Efeitos marginais significativos a $1 \%$ e $5 \%$, para cada proxy de utilização

cons_med_g - $\mathrm{N}^{\circ}$ de consultas com médico de medicina geral e familiar nas últimas 4 semanas; cons_espec - $\mathrm{N}^{\circ}$ de consultas com médico de uma especialidade hospitalar nas últimas 4 semanas; cons_total $-\mathrm{N}^{\circ}$ de consultas com médico de medicina geral e familiar ou médico de uma especialidade hospitalar últimas 4 semanas

residir em zona pouco povoada) bem como o maior uso no caso de famílias monoparentais. Estar empregado reduz a utilização, o mesmo acontecendo no caso de quem não beneficia de nenhum subsistema ou seguro de saúde (no caso da utilização de consultas de especialidades hospitalares e totais). Em termos de regiões NUTS II, e realçando os efeitos estatisticamente significativos, o Algarve e Madeira apresentam menor utilização do que o Norte e o contrário ocorre com Lisboa.

Observando agora, na Tabela 2, a distribuição da utilização de consultas, constata-se que a utilização de consultas de medicina geral e familiar está desproporcionalmente concentrada nos mais pobres, enquanto as consultas de especialidades hospitalares se encontram concentradas nos mais ricos embora a concentração seja de menor amplitude neste caso. Os dois efeitos combinados conduzem a uma utilização de consultas totais quase igualmente distribuída. Embora o índice de concentração seja estatisticamente significativo, o seu valor é muito próximo de zero.

Tabela 2 - Índice de concentração para o número efetivo de consultas

\begin{tabular}{lc}
\hline Tipo de consulta & Índice de concentração \\
\hline cons_med_g & $-0,04688^{* * *}$ \\
cons_espec & $0,016291^{* * *}$ \\
cons_total & $-0,0091^{* * *}$ \\
\hline$* * * 0<01 \cdot * * p<0,05 \cdot{ }^{*} p<0,1$ &
\end{tabular}

Contudo, para a análise da equidade há que confrontar o uso com a necessidade. Assim, a Tabela 3 apresenta os resultados para o índice de iniquidade horizontal.

Em traços gerais, não podemos concluir pela existência de iniquidade na utilização de consultas de medicina geral e familiar (índice praticamente nulo e não significativo). Por outro lado, nas consultas de especialidades hospitalares e nas consultas totais há evidência de existência de iniquidade horizontal a favor dos mais ricos (índices positivos e estatisticamente significativos a $1 \%$ ).

\section{DISCUSSÃO}

Com o presente estudo pretendíamos monitorizar o desempenho de Portugal no que diz respeito ao princípio de igual utilização de consultas médicas para igual necessidade. Pela análise do índice de iniquidade horizontal, concluímos que no caso de consultas de medicina geral e familiar não se pode excluir a verificação dos objetivos de equidade relacionada com o rendimento; contudo, persiste

Tabela 3 - Índices de concentração para a utilização, padronizada pela necessidade

\begin{tabular}{lc}
\hline Tipo de consulta & Índice de iniquidade horizontal \\
\hline cons_med_g & 0,00232 \\
cons_espec & $0,066897^{* * *}$ \\
cons_total & $0,053554^{* * *}$ \\
\hline
\end{tabular}

${ }^{* * *} p<0,01 ;{ }^{* *} p<0,05 ;{ }^{*} p<0,1$ 
iniquidade favorável aos mais ricos no caso de consultas de especialidades hospitalares. Porém, comparando com estudos prévios, a evidência sugere a ocorrência de progressos importantes conforme mostra a Fig. 2. O índice para consultas de medicina geral e familiar manteve-se estável durante cerca de duas décadas e com um valor próximo de zero. O índice para consultas de especialidades hospitalares sofreu um aumento entre 1996 e 2000, verificando a partir de então uma queda acentuada, atingindo em 2014 cerca de um terço do seu valor em 2000. Outro estudo que usou dados de 1995 a $2001,{ }^{10}$ cujos resultados não estão reportados na Fig. 2, encontrou valores similares aos de 2000 (índice de iniquidade horizontal igual a 0,018 e 0,199 , para consultas de medicina geral e familiar e de especialidades hospitalares, respetivamente).

Em termos de consultas totais, verifica-se a existência de iniquidade favorável aos mais ricos mas de menor intensidade, dada a possibilidade de existência de um efeito de compensação entre tipos de consultas. Apenas um estudo anterior reporta resultados para o índice de iniquidade horizontal para consultas totais, ${ }^{9}$ sendo de 0,068 , para o ano 2000. De qualquer modo, usar o índice de iniquidade relativo a consultas totais para avaliar a equidade na utilização de consultas implica assumir indiferença entre uma consulta de uma especialidade hospitalar e uma consulta de medicina geral e familiar. Tal poderá ser mais aceitável nuns casos do que noutros. As variáveis de necessidade disponíveis no INS 2014 e usadas neste estudo, como em estudos similares, não nos permitem distinguir necessidades mais específicas e que porventura justificassem o uso de consultas de especialidade. Não se pode excluir a possibilidade dos resultados obtidos ao longo dos anos para consultas de especialidades hospitalares refletirem em parte uma sobreutilização deste tipo de cuidados. Tendo em conta que a maior parte das consultas de especialidades hospitalares em Portugal tem lugar nos cuidados ambulatórios privados, ${ }^{16,17}$ quem tem acesso facilitado a estes cuidados, sem passar pelo filtro da referenciação dos cuidados primários, poderá utilizá-los mesmo em casos em que tal não se justifique por razões clínicas.

Efetivamente, as diferenças encontradas, na utilização padronizada, entre grupos de rendimento, podem decorrer da desigual distribuição de diversos fatores, quer do lado da procura quer do lado da oferta. Confrontando os valores obtidos para o índice com os resultados da análise de regressão, em que o rendimento não se revela uma variável significativa na explicação da utilização de consultas, outros fatores parecem estar a causar a iniquidade observada entre grupos de rendimento. A evidência sugere que um desses fatores é a educação, cujo impacto na utilização se aproxima do efeito de variáveis de necessidade como o estado de saúde autoavaliado e a existência de limitações na realização de atividades diárias. Não sendo a educação uma variável de necessidade, esta situação configura iniquidade na utilização de consultas médicas. Os nossos resultados apontam ainda para a existência de iniquidade geográfica (favorável à região de Lisboa e zonas urbanas em geral, e desfavorável ao Algarve e Madeira) e iniquidade em termos de cobertura de seguros (neste caso desfavorável a quem tem apenas a cobertura do Serviço Nacional de Saúde).

O impacto negativo (menor utilização) e significativo que estar empregado representa pode estar relacionado possivelmente com questões de custos de oportunidade e disponibilidade para consultar um médico.

Relativamente às variáveis de necessidade, o seu

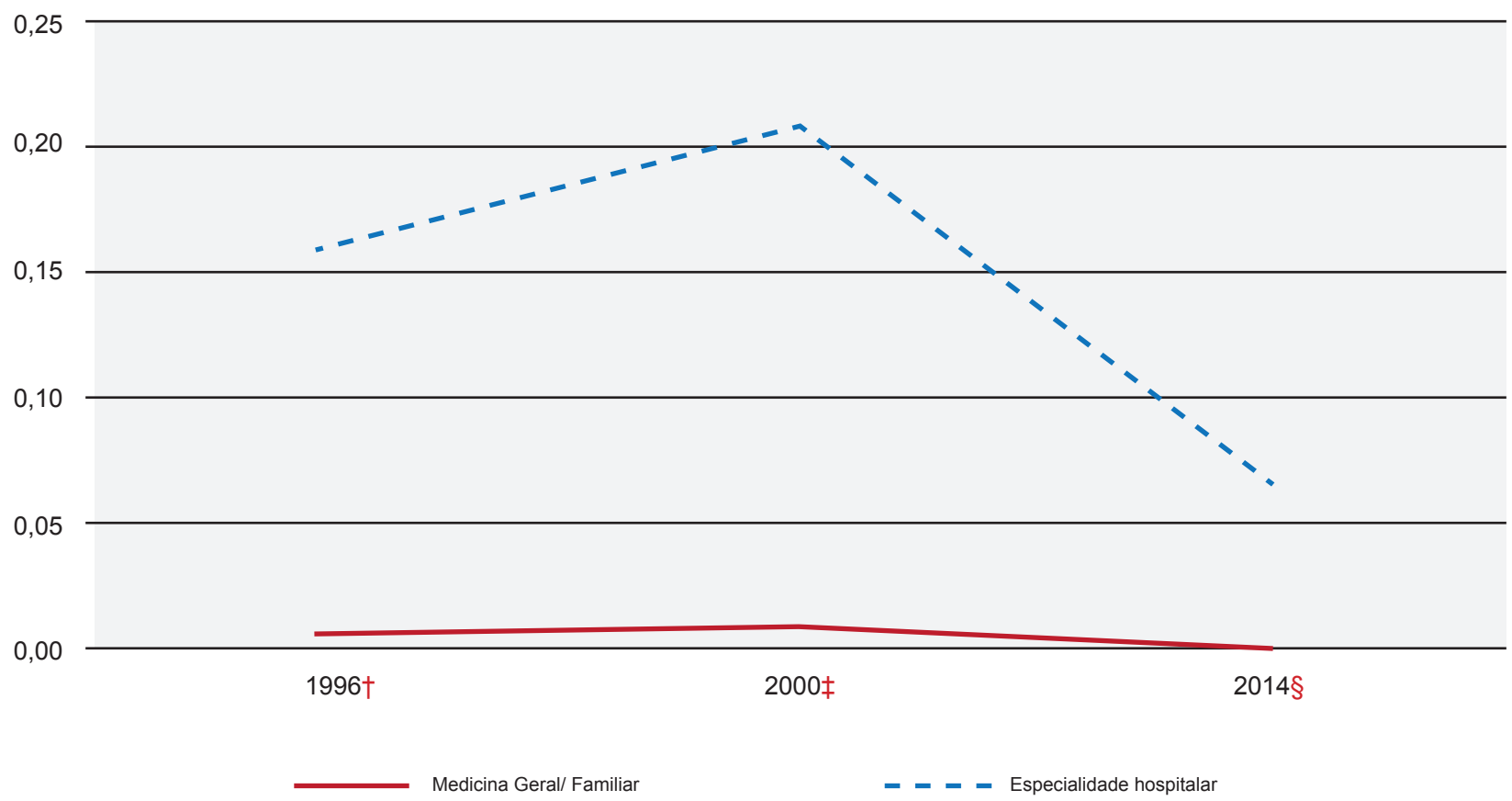

Figura 2 - Índice de iniquidade horizontal para consultas de medicina geral e familiar e de especialidade hospitalar: evolução $1996-2014$ † ref. 8; † ref. 9; § cálculos próprios 
impacto é compatível com a noção de utilização de acordo com a necessidade. Pelos resultados da Tabela 1, observa-se que a magnitude do impacto das variáveis estado de saúde bom ou muito bom e estado de saúde razoável é superior para consultas de especialidades hospitalares em comparação com consultas de medicina geral e familiar. Já no caso de problemas de saúde prolongados ou doenças crónicas, estas variáveis não são significativas para explicar a utilização de consultas de especialidades hospitalares. Estas variáveis poderão estar a captar diferentes tipos de necessidade, relacionados com situações de doença aguda versus doença crónica.

Apelando à terminologia de Andersen, ${ }^{3}$ obtivemos, como esperado, evidência do impacto de variáveis de necessidade. Em termos das variáveis de capacitação, o rendimento não se revelou pertinente em si mesmo enquanto a cobertura de cuidados oferecida pelo SNS se mostrou desfavorável face às situações em que existiam seguros adicionais. A variável educação destacou-se pelo seu impacto considerável sendo que este resultado tanto pode refletir o papel de fatores de capacitação (para, por exemplo, navegar os serviços de saúde) como de fatores predisponentes. De facto, o valor que os indivíduos atribuem à própria saúde tende a afetar a procura de cuidados. Além do mais, existindo evidência em Portugal de uma associação positiva entre educação e literacia em saúde,,$^{18}$ o impacto da variável educação poderá ainda estar relacionado com o benefício percebido dos cuidados de saúde.

Conforme atrás mencionado, uma limitação da nossa análise diz respeito à dificuldade de identificar necessidades de cuidados de saúde que exijam de facto o recurso a uma consulta de uma especialidade hospitalar por oposição a uma de medicina geral e familiar. Adicionalmente, os dados disponíveis também não permitem fazer qualquer distinção em termos da qualidade dos cuidados recebidos nem em termos da utilização atempada e dentro dos tempos clinicamente aconselháveis. É necessário ter ainda em conta que a presente análise ignora o ónus financeiro suportado pelas famílias com a utilização de cuidados de saúde. Assim, uma menor desigualdade na utilização pode emergir à custa de grande sacrifício por parte das famílias mais pobres. Por outro lado, este estudo apenas considerou as consultas médicas, ficando fora da análise a utilização de cuidados hospitalares. No entanto, existe evidência que os mais pobres por vezes recorrem aos cuidados hospitalares, nomeadamente urgências, como substitutos de consultas de medicina geral e familiar ou de especialidade hospitalar. ${ }^{12}$

Por fim, coloca-se a questão fundamental sobre se, e em que medida, as iniquidades na utilização de cuidados de saúde se convertem em iniquidades em saúde. ${ }^{9}$ Embora exista evidência que o acesso não realizado (necessidades de cuidados de saúde não satisfeitas) conduz a uma deterioração subsequente do estado de saúde do indivíduo, ${ }^{19}$ as determinantes sociais da saúde vão muito além dos cuidados de saúde. ${ }^{20} \mathrm{Na}$ verdade, a influência destas outras determinantes é de tal ordem que deverá ultrapassar o efeito dos fatores ao alcance da medicina. ${ }^{21}$

Esta possível associação entre iniquidade na utilização de cuidados de saúde e iniquidade em saúde é ainda mais pertinente em contextos de recessão económica. Um estudo para Espanha revelou que nem o acesso universal aos cuidados de saúde impediu a crise económica e financeira de afetar de modo diferenciado os diferentes grupos de rendimento (no caso dos mais pobres o efeito restritivo sobre a utilização manifestou-se essencialmente ao nível das consultas de especialidades hospitalares e hospitalizações embora o seu uso de urgências e consultas de medicina geral e familiar tenha aumentado). ${ }^{22}$ Um outro estudo, para Portugal, conclui pela existência de uma associação entre os cortes orçamentais decorrentes da implementação do acordo com a troika e iniquidades crescentes no acesso aos cuidados de saúde por parte dos idosos. ${ }^{23}$ Contudo, parece ainda ser demasiado cedo para se poder avaliar completamente o impacto da austeridade sobre a equidade em saúde ${ }^{24}$ e deverá levar o seu tempo para que os efeitos de longo prazo sobre esta se tornem visíveis e mensuráveis. ${ }^{25}$

\section{CONCLUSÃO}

O princípio de igual utilização de cuidados de saúde para igual necessidade continua a figurar entre os objetivos da política de saúde em Portugal. Estudos prévios, com base em dados até 2001 , colocavam o país ainda longe destes objetivos no que diz respeito às consultas médicas. Com o presente trabalho procurámos avaliar a situação com base em dados mais atualizados.

Os resultados obtidos apontam no sentido de um notável progresso em termos de equidade relacionada com o rendimento na utilização de consultas médicas. Persiste iniquidade, favorável aos mais ricos, no uso de consultas de especialidades hospitalares, mas a sua magnitude diminuiu consideravelmente.

Não obstante este avanço, os resultados da análise de regressão mostram que vários fatores, não relacionados com a necessidade, ainda continuam a afetar a utilização de consultas médicas. Mais do que o rendimento, a educação assume um papel fundamental e, em menor escala, verificam-se iniquidades geográficas e na cobertura de seguros de saúde. Assim, a evidência sugere que o país conseguiu alguns progressos, e isto apesar da crise económica e financeira que o afetou recentemente, mas permanecem desafios no sentido de assegurar a equidade na utilização de cuidados de saúde. Pelos nossos resultados, responder a estes desafios passa sobretudo por políticas públicas em várias áreas, com maior ou menor abrangência, quer em termos dos grupos populacionais quer em termos geográficos, no sentido de garantir que quem tem necessidade acede aos serviços de saúde. Aos profissionais de saúde competirá sobretudo assegurar que, uma vez chegados aos serviços de saúde, os cidadãos são tratados de modo equitativo, tendo em conta a sua necessidade e independentemente de outras quaisquer características. Cabe ainda aos profissionais de saúde avaliar sobre a adequação dos cuidados de saúde que constituem 
a norma. Isto é, os estudos sobre a equidade na utilização de cuidados de saúde olham para os desvios face à norma. No entanto, nada se pode concluir sobre a utilização média de cuidados, podendo esta até ser excessiva ou, ao invés, insuficiente.

\section{PROTECÇÃO DE PESSOAS E ANIMAIS}

Os autores declaram que os procedimentos seguidos estavam de acordo com os regulamentos estabelecidos pelos responsáveis da Comissão de Investigação Clínica e Ética e de acordo com a Declaração de Helsínquia da Associação Médica Mundial.

\section{REFERÊNCIAS}

1. O'Donnell O, van Doorslaer E, Wagstaff A, Lindelow M. Analyzing health equity using survey data: a guide to techniques and their implementation. Washington: The World Bank; 2008.

2. Direção Geral de Saúde/Ministério da Saúde. Plano Nacional de Saúde - revisão e extensão a 2020. Lisboa: DGS; 2015.

3. Andersen RM. Revisiting the behavioral model and access to medical care: does it matter? J Health Soc Behav. 1995;36:1-10.

4. Levesque JF, Harris MF, Russell G. Patient-centred access to health care: conceptualising access at the interface of health systems and populations. Int J Equity Health. 2013;12:18.

5. Direção Geral de Saúde/Ministério da Saúde. Plano Nacional de Saúde 2012-2016. Lisboa: DGS; 2013.

6. Wagstaff A, van Doorslaer E. Equity in Health care finance and delivery. In: Culyer AJ, Newhouse JP, editores. Handbook of health economics, New York: Elsevier; 2000. p.1803-62.

7. Furtado C, Pereira J Equidade e acesso aos cuidados de saúde. Lisboa: Alto Comissariado da Saúde; (2010). [consultado 2019 abr 16]. Disponível em: https://www.researchgate.net/publication/268414998_ Equidade_e_Acesso_aos_Cuidados_de_Saude.

8. van Doorslaer E, Koolman X, Jones AM. Explaining income-related inequalities in doctor utilization in Europe. Health Econ. 2004;13:629_ 47.

9. van Doorslaer E, Masseria C. Income-related inequality in the use of medical care in 21 OECD countries. Paris: OECD; 2004.

10. d'Uva TB, Jones AM, van Doorslaer E. Measurement of horizontal inequity in health care utilisation using European panel data. J Health Econ. 2009;28:280-9.

11. Lourenço Ó, Quintal C, Ferreira PL, Barros PP. A equidade na utilização de cuidados de saúde em Portugal: uma avaliação baseada em modelos de contagem. Notas Econ. 2007;6-27.

12. Or Z, Jusot F, Yilmaz E. Impact of health care system on socioeconomic inequalities in doctor use for the European Union Working Group on Socioeconomic Inequalities in Health. IRDES Working paper 17; 2008

13. Tavares LP, Zantomio, F. Inequity in healthcare use among older people after 2008: the case of southern European countries. Health Policy. 2017;121:1063-71.

\section{CONFIDENCIALIDADE DOS DADOS}

Os autores declaram ter seguido os protocolos do seu centro de trabalho acerca da publicação de dados.

\section{CONFLITOS DE INTERESSE}

Os autores declaram não ter conflitos de interesse relacionados com o presente trabalho.

\section{FONTES DE FINANCIAMENTO}

Este trabalho não recebeu qualquer tipo de suporte financeiro de nenhuma entidade no domínio público ou privado.

14. Instituto Nacional de Estatística. INE (2016): Inquérito Nacional de Saúde 2014. [consultado 2019 abr 24]. Disponível em: https://www.ine.pt/ xportal/xmain?xpid=INE\&xpgid=ine_publicacoes\&PUBLICACOESpub_ boui=263714091\&PUBLICACOESmodo=2.

15. Kakwani NC, Wagstaff $A$, van Doorslaer E. Socioeconomic inequalities in health: measurement, computation and statistical inference. J Econom. 1997;77:87-104.

16. Thomson S, Foubister T, Mossialos E. Financing health care in the European Union - challenges and policy responses. Copenhaga: WHO; 2009.

17. Simões J, Augusto GF, Fronteira I, Hernández-Quevedo C. Portugal: health system review. Health Syst Transit. 2017;19:1-184.

18. Espanha R, Ávila P, Mendes R. Literacia em saúde em Portugal. Lisboa: Fundação Calouste Gulbenkian; 2016.

19. Gibson G, Grignon M, Hurley J, Wang L. Here comes the SUN: selfassessed unmet need, worsening health outcomes, and health care inequity. Health Econ. 2019;28:727-35.

20. Marmot M, Allen J, Bell R, Bloomer E, Goldblatt P. WHO European review of social determinants of health and the health divide. Lancet. 2012;380;1011-29.

21. Carrapato $P$, Correia $P$, Garcia B. Determinante da saúde no Brasil: a procura da equidade na saúde. Saúde Soc. 2017;26:676-89.

22. Abásolo I, Saez M, López-Casasnovas G. Financial crisis and incomerelated inequalities in the universal provision of a public service: the case of healthcare in Spain. Int J Equity Health. 2017;16:134.

23. Doetsch J, Pilot E, Santana P, Krafft T. Potential barriers in healthcare access of the elderly population influenced by the economic crisis and the troika agreement: a qualitative case study in Lisbon, Portugal. Int $\mathrm{J}$ Equity Health. 2017;16:184.

24. Ruckert $A$, Labonte $R$. Health inequities in the age of austerity: the need for social protection policies. Soc Sci Med. 2017;187:306-11.

25. Bambra C, Garthwaite K, Copeland A, Barr B. All in it together? Health inequalities, austerity, and the "Great Recession". In: Smith KE, Bambra C, Hill SE, editors. Health inequalities. Oxford: Oxford University Press; 2015. p. 164-76. 